International Mathematical Forum, 2, 2007, no. 41, 2045 - 2054

\title{
Record Values from the Modified Weibull Distribution and Applications
}

\author{
Khalaf S. Sultan \\ Department of Statistics and Operations Research \\ College of Science, King Saud University \\ P.O. Box 2455, Riyadh 11451, Saudi Arabia
}

\begin{abstract}
In this paper, we establish some new recurrence relations between the single moments of record values from the modified Weibull distribution as well as between the double moments. Next, we show that the results for the record values from the Weibull distribution obtained by Balakrishnan and Chan (1993) can be derived from our results as special cases. In addition, we deduce some new recurrence relations of the single and double moments of record values from log-gamma distribution. Further, we use the established recurrence relations to calculate the mean, variance and the product moments. Finally, we suggest two applications.
\end{abstract}

Mathematics Subject Classification: 62G99, 62E12

Keywords: Upper record values; single moments, double moments, recurrence relations, best linear unbiased estimate and characterization

\section{Introduction}

The modified Weibull distribution was proposed by Lai, Xie and Murthy (2003) as a new lifetime distribution. They have shown the capability of the model for modeling a bathtub-shaped hazard-rate function. In addition, they characterized the model through the Weibull plot paper. Further, they have shown that the modified Weibull model compares well with other competing models to fit data that exhibit a bathtub-shaped hazard-rate function.

A random variable $X$ is said to have the modified Weibull distribution (MWD) if its probability density function (pdf) is given by

$$
f(x)=a(b+\lambda x) x^{b-1} e^{\lambda x} \exp \left(-a x^{b} e^{\lambda x}\right), a, \lambda>0, b \geq 0, x>0
$$


while the cumulative distribution function (cdf) is given by

$$
F(x)=1-\exp \left(-a x^{b} e^{\lambda x}\right), a, \lambda>0, b \geq 0, x>0 .
$$

It is easy to derive the relation between the pdf and cdf of MWD given in (1.1) and (1.2), respectively, as

$$
\frac{f(x)}{1-F(x)}=-\log (1-F(x)) \frac{b+\lambda x}{x} .
$$

The MWD given in (1.1) is a limiting distribution of the beta-integrated model [see Moore and Xie (1988)] and it is related to the following well-know distributions:

1. At $\lambda=0$, MWD reduces to the two-parameter Weibull distribution.

2. At $b=0$, MWD reduces to a type I extreme-value distribution and is also known as a log-gamma distribution or log-Weibull distribution [see Johnson, Kotz and Balakrishnan (1995)].

Record values arise naturally in many real life applications involving data relating to weather, sports, economics and life testing studies. Many authors have studied the record values and associated statistics; for example, see Chandler (1952), Ahsanullah (1980, 1988, 1990, 1993, 1995), and Arnold, Balakrishnan and Nagaraja (1992, 1998). Balakrishnan and Chan (1993), and Balakrishnan, Ahsanullah and Chan (1995) have discussed some inferential methods for exponential, Gumbel, Weibull and logistic distributions, respectively. Sultan and Moshref (2000) have obtained the best linear unbiased estimates for the location and scale parameters of record values from the generalized Pareto distribution. Maximum likelihood estimates of parameters for some useful distributions, including one and two parameter exponential, one and two parameter uniform, normal, logistic and Gumbel distributions are discussed in Arnold, Balakrishnan and Nagaraja (1998). Balakrishnan and Ahsanullah (1994) have established some recurrence relations satisfied by the single and double moments of upper record values from the standard form of the generalized Pareto distribution.

Let $X_{U(1)}, X_{U(2)}, \ldots, X_{U(n)}$ denote the first $n$ upper record values arising from a sequence $\left\{X_{i}\right\}$ of iid MWD variables. Then the $p d f$ of $X_{U(n)}$ is given by [see Arnold, Balakrishnan and Nagaraja (1998)]

$$
f_{n}(x)=\frac{1}{\Gamma(n)}\{-\log [1-F(x)]\}^{n-1} f(x), x>0, n=1,2, \ldots,
$$

while the joint $p d f$ of $X_{U(m)}$ and $X_{U(n)}, m<n$ is given by

$f_{m, n}(x, y)=\frac{1}{\Gamma(m) \Gamma(n-m)}[-\log (1-F(y))+\log (1-F(x))]^{n-m-1}$ 


$$
\times[-\log (1-F(x))]^{m-1} \frac{f(x) f(y)}{1-F(x)}, 0<x<y<\infty, m=1,2, \ldots, m<n .
$$

In the following section, we establish some new recurrence relations between the different moments of record values from MWD.

\section{Moments}

Let $X_{U(1)}, X_{U(2)}, \ldots X_{U(n)}$ be the first $n$ upper record values from the MWD given in(1.1), then the single and double moments of the upper record values are given by

$$
\mu_{n}^{(r)}=E\left(X^{r}\right)=\int_{0}^{\infty} x^{r} f_{n}(x) d x, \quad r=0,1,2, \ldots
$$

and

$$
\begin{array}{r}
\mu_{m, n}^{(r, s)}=E\left(X^{r} Y^{s}\right)=\int_{0}^{\infty} \int_{0}^{y} x^{r} y^{s} f_{m, n}(x, y) d x d y, \quad 0<x<y<\infty \\
m=1,2, \ldots, m<n, r, s=0,1,2, \ldots
\end{array}
$$

where $f_{n}($.$) and f_{m, n}(.,$.$) are given, respectively, by (1.4) and (1.5). The single$ and double moments of upper record values from MWD given in (2.1) and (2.2), respectively, satisfy the following recurrence relations.

\section{Relation 2.1}

For $n \geq 1$ and $r=0,1,2, \ldots$, the single moments of upper record values from MWD defined in (2.1) satisfy the recurrence relation

$$
\mu_{n}^{(r)}=\frac{n b}{r}\left[\mu_{n+1}^{(r)}-\mu_{n}^{(r)}\right]+\frac{n \lambda}{r+1}\left[\mu_{n+1}^{(r+1)}-\mu_{n}^{(r+1)}\right]
$$

\section{Proof}

From (1.3) and (2.1), the single moments of record values from MWD can be written as

$$
\mu_{n}^{(r)}=\frac{1}{\Gamma(n)}\left[b I_{r-1}+\lambda I_{r}\right]
$$

where

$$
I_{r}=\int_{0}^{\infty} x^{r}[-\log (1-F(x))]^{n}(1-F(x)) d x,
$$


upon integrating (2.5) by parts treating $x^{r}$ for integration and the rest of the integrand for differentiation, we obtain

$$
I_{r}=\frac{\Gamma(n+1)}{r+1} \mu_{n+1}^{(r+1)}-\frac{\Gamma(n+1)}{r+1} \mu_{n}^{(r+1)}
$$

where $\Gamma($.$) is the gamma function.$

Similarly, we write

$$
I_{r-1}=\frac{\Gamma(n+1)}{r} \mu_{n+1}^{(r)}-\frac{\Gamma(n+1)}{r} \mu_{n}^{(r)} .
$$

From (2.4), (2.6) and (2.7), then (2.3) is proved.

\section{Relation 2.2}

For $m \geq 1$ and $r, s=0,1,2, \ldots$, the double moments of upper record values from MWD defined in (2.2) satisfy the recurrence relation

$$
\mu_{m, m+1}^{(r, s)}=\frac{m b}{r}\left[\mu_{m+1}^{(r+s)}-\mu_{m, m+1}^{(r, s)}\right]+\frac{m \lambda}{r+1}\left[\mu_{m}^{(r+s+1)}-\mu_{m, m+1}^{(r+1, s)}\right]
$$

and for $1 \leq m \leq n-1$ and $r, s=0,1,2, \ldots$, we have

$$
\mu_{m, n}^{(r, s)}=\frac{m b}{r}\left[\mu_{m+1, m}^{(r, s)}-\mu_{m, n}^{(r, s)}\right]+\frac{m \lambda}{r+1}\left[\mu_{m+1, n}^{(r+1, s)}-\mu_{m, n}^{(r+1, s)}\right] .
$$

\section{Proof}

From (2.2), the double moments of record values from MWD can be written as

$$
\mu_{m, n}^{(r, s)}=\frac{1}{\Gamma(m) \Gamma(n-m)} \int_{0}^{\infty} y^{s} f(y) I(y) d y
$$

where

$$
\begin{aligned}
I(y) & =\int_{0}^{y} x^{r}[-\log (1-F(x))]^{m-1} \\
& \times[-\log (1-F(y))+\log (1-F(x))]^{n-m-1} \frac{f(x)}{1-F(x)} d x,
\end{aligned}
$$

1. For $n=m+1$ and by using (1.3), we can write (2.11) as

$$
I(y)=b T_{r-1}+\lambda T_{r}
$$


where

$$
T_{r}=\int_{0}^{y} x^{r}[-\log (1-F(x))]^{m} d x,
$$

which upon integrating by parts gives

$$
\begin{aligned}
T_{r} & =\frac{y^{r+1}}{r+1}[-\log (1-F(y))]^{m} \\
& -\frac{m}{r+1} \int_{0}^{y} x^{r+1}[-\log (1-F(x))]^{m-1} \frac{f(x)}{1-F(x)} d x
\end{aligned}
$$

Similarly, we write

$$
\begin{aligned}
T_{r-1} & =\frac{y^{r}}{r}[-\log (1-F(y))]^{m} \\
& -\frac{m}{r} \int_{0}^{y} x^{r}[-\log (1-F(x))]^{m-1} \frac{f(x)}{1-F(x)} d x .
\end{aligned}
$$

From (2.10), (2.12), (2.13) and (2.14), then (2.8) is proved.

2. For $1 \leq m \leq n-1$ and by making use of (1.3), we write (2.11) as

$$
I(y)=b Q_{r-1}+\lambda Q_{r}
$$

where

$$
\begin{aligned}
Q_{r} & =\frac{n-m-1}{r+1} \int_{0}^{y} x^{r+1}[-\log (1-F(x))]^{m} \\
& \times[-\log (1-F(y))+\log (1-F(x))]^{n-m-2} \frac{f(x)}{1-F(x)} d x \\
& -\frac{m}{r+1} \int_{0}^{y} x^{r+1}[-\log (1-F(x))]^{m-1} \\
& \times[-\log (1-F(y))+\log (1-F(x))]^{n-m-1} \frac{f(x)}{1-F(x)} d x
\end{aligned}
$$

and

$$
\begin{aligned}
Q_{r-1} & =\frac{n-m-1}{r} \int_{0}^{y} x^{r}[-\log (1-F(x))]^{m} \\
& \times[-\log (1-F(y))+\log (1-F(x))]^{n-m-2} \frac{f(x)}{1-F(x)} d x \\
& -\frac{m}{r} \int_{0}^{y} x^{r}[-\log (1-F(x))]^{m-1} \\
& \times[-\log (1-F(y))+\log (1-F(x))]^{n-m-1} \frac{f(x)}{1-F(x)} d x(2.17)
\end{aligned}
$$

From (2.15), (2.16) and (2.17), then (2.9) is proved. 
The recurrence relations for the single and double moments of record values can be used to calculate the different moments for any order and sample size in a simple regressive manner. The recurrence relations reduce the round off error for calculating the moments compare with the numerical integration techniques. When we use the recurrence relations to calculate the moments, we need only few initial moments to be numerically calculated.

\section{Example}

To calculate the moments $\mu_{n}^{(r)}, r=1,4$ and $n=1,10$, we need only $\mu_{n}^{(1)}, n=1,3, \mu_{1}^{(2)}, \mu_{1}^{(3)}$ and $\mu_{1}^{(4)}$ to be calculated by using a numerical integration technique. Next, we use the recurrence relations to calculate the rest of the required moments. Table 1 displays the means and variances of the upper record values up to $n=10$, while Table 2 shows the product moments.

Table 1: Mean and variances of record values when $\lambda=0.1$

\begin{tabular}{lllll}
\hline & $\mathrm{b}=1$ & & $\mathrm{~b}=0.4$ & \\
\hline$n$ & Mean & Variance & Mean & Variance \\
\hline 1 & 0.8556 & 0.5658 & 1.1213 & 2.7199 \\
2 & 1.6029 & 0.8944 & 2.7502 & 5.8093 \\
3 & 2.2664 & 1.0871 & 4.4427 & 7.5981 \\
4 & 2.8632 & 1.1990 & 6.0154 & 8.1858 \\
5 & 3.4058 & 1.2612 & 7.4226 & 8.0949 \\
6 & 3.9035 & 1.2922 & 8.6710 & 7.7066 \\
7 & 4.3635 & 1.3033 & 9.7820 & 7.2217 \\
8 & 4.7911 & 1.3013 & 10.7778 & 6.7309 \\
9 & 5.1910 & 1.2910 & 11.6776 & 6.2704 \\
10 & 5.5665 & 1.2752 & 12.4972 & 5.8516 \\
\hline
\end{tabular}


Table 2: Double moments of record values when $\lambda=0.1$

\begin{tabular}{lllllllll}
\hline \hline$m$ & $n$ & $\mu_{m, n}$ & $m$ & $n$ & $\mu_{m, n}$ & $m$ & $n$ & $\mu_{m, n}$ \\
\hline$b=1.0$ & & & & & & & & \\
1 & 2 & 1.8731 & 1 & 7 & 4.0554 & 3 & 9 & 12.4445 \\
1 & 3 & 2.3899 & 2 & 7 & 7.5676 & 4 & 9 & 15.6880 \\
2 & 3 & 4.4362 & 3 & 7 & 10.6645 & 5 & 9 & 18.6268 \\
1 & 4 & 2.8592 & 4 & 7 & 13.4348 & 6 & 9 & 21.3147 \\
2 & 4 & 5.3189 & 5 & 7 & 15.9420 & 7 & 9 & 23.7925 \\
3 & 4 & 7.4760 & 6 & 7 & 18.2329 & 8 & 9 & 26.0917 \\
1 & 5 & 3.2893 & 1 & 8 & 4.4003 & 1 & 10 & 5.0291 \\
2 & 5 & 6.1276 & 2 & 8 & 8.2155 & 2 & 10 & 9.3967 \\
3 & 5 & 8.6229 & 3 & 8 & 11.5827 & 3 & 10 & 13.2566 \\
4 & 5 & 10.8497 & 4 & 8 & 14.5972 & 4 & 10 & 16.7158 \\
1 & 6 & 3.6864 & 5 & 8 & 17.3272 & 5 & 10 & 19.8512 \\
2 & 6 & 6.8741 & 6 & 8 & 19.8230 & 6 & 10 & 22.7200 \\
3 & 6 & 9.6814 & 7 & 8 & 22.1229 & 7 & 10 & 25.3652 \\
4 & 6 & 12.1900 & 1 & 9 & 4.7240 & 8 & 10 & 27.8204 \\
5 & 6 & 14.4585 & 2 & 9 & 8.8236 & 9 & 10 & 30.1122 \\
$b=0.4$ & & & & & & & & \\
1 & 2 & 5.8535 & 1 & 7 & 12.581 & 3 & 9 & 55.814 \\
1 & 3 & 7.5356 & 2 & 7 & 30.304 & 4 & 9 & 74.971 \\
2 & 3 & 17.6099 & 3 & 7 & 48.252 & 5 & 9 & 91.942 \\
1 & 4 & 9.0241 & 4 & 7 & 64.607 & 6 & 9 & 106.896 \\
2 & 4 & 21.3622 & 5 & 7 & 79.035 & 7 & 9 & 120.140 \\
3 & 4 & 33.5266 & 6 & 7 & 91.708 & 8 & 9 & 131.972 \\
1 & 5 & 10.3436 & 1 & 8 & 13.543 & 1 & 10 & 15.233 \\
2 & 5 & 24.6844 & 2 & 8 & 32.713 & 2 & 10 & 36.933 \\
3 & 5 & 39.0047 & 3 & 8 & 52.208 & 3 & 10 & 59.125 \\
4 & 5 & 51.9060 & 4 & 8 & 70.031 & 4 & 10 & 79.503 \\
1 & 6 & 11.5214 & 5 & 8 & 85.792 & 5 & 10 & 97.580 \\
2 & 6 & 27.6445 & 6 & 8 & 99.662 & 6 & 10 & 113.524 \\
3 & 6 & 43.8793 & 7 & 8 & 111.935 & 7 & 10 & 127.655 \\
4 & 6 & 58.6046 & 1 & 9 & 14.423 & 8 & 10 & 140.285 \\
5 & 6 & 71.5501 & 2 & 9 & 34.912 & 9 & 10 & 151.674 \\
\hline & & & & & & & &
\end{tabular}




\section{Applications}

In this section, we suggest some applications based on the moments discussed in Section 2.

1. Estimation: The moments of record values given in Section 2 can be used to obtain the best linear unbiased estimate of the scale parameters of the modified Weibull

$$
f(x)=\frac{1}{\sigma}\left(b+\lambda \frac{x}{\sigma}\right)\left(\frac{x}{\sigma}\right)^{b-1} e^{\lambda \frac{x}{\sigma}} \exp \left(-a(x / \sigma)^{b} e^{\lambda x / \sigma}\right), a, \lambda>0, b \geq 0, x>0 .
$$

Some work of this nature have been done by Sultan and Moshref (2002).

2. Characterization: The modified Weibull distribution given in (1.2) can be characterized by using the conditional expectation of record values as follows

\section{Theorem 1}

The values $x$ and $y$ represent, respectively, the $m$-th and $(m+1)$-th record values from the modified Weibull distribution iff

$$
E\left[\exp \left(-y^{b} e^{\lambda y}\right) \mid X=x\right]=\frac{a}{1+a} \exp \left(-x^{b} e^{\lambda x}\right)
$$

\section{Proof}

The proof can be easily done by using the definition of the conditional record values.

Theorem 3.1 can be used to characterize the Weibull and log-gamma distribution by substituting $\lambda=0$ and $b=0$ in (3.1).

\section{Concluding Remarks}

Some new recurrence relation between the single and product moments of record values from the modified Weibull distribution are established. In addition the single and product moments are calculated for some choices the the parameters. Further, the conditional expectation of function of record values is used to characterize the distribution. The relation between our results and some other results in literature are listed below:

1. When $\lambda=0$, Relation 2.1 reduces to recurrence relation of the single and double moments of record values from Weibull distribution obtained by Balakrishnan and Chan (1993). 
2. When $b=0$, we deduce

$$
\begin{gathered}
\mu_{n}^{(r)}=\frac{n \lambda}{r+1}\left[\mu_{n+1}^{(r+1)}-\mu_{n}^{(r+1)}\right], \\
\mu_{m, m+1}^{(r, s)}=\frac{m \lambda}{r+1}\left[\mu_{m}^{(r+s+1)}-\mu_{m, m+1}^{(r+1, s)}\right] ;
\end{gathered}
$$

and for $1 \leq m \leq n-1$ and $r, s=0,1,2, \ldots$, we have

$$
\mu_{m, n}^{(r, s)}=\frac{m \lambda}{r+1}\left[\mu_{m+1, n}^{(r+1, s)}-\mu_{m, n}^{(r+1, s)}\right] .
$$

which are the recurrence relations of the single and double moments of upper record values from log-gamma distribution.

3. the single and product moment can be given in Tables 1 and 2 can be used to calculate the variance covariance matrix or record values from MWD which unable us to obtain the best linear unbiased estimates for the location and scale parameters of the MWD.

Acknowledgements: The author would like to thank the research center, College of Science, King Saud University for funding this project.

\section{REFERENCES}

[1] M. Ahsanullah, Linear prediction of record values for the two parameter exponential distribution, Ann. Inst. Statist. Math., 2(1980), 363-368.

[2] M. Ahsanullah, Introduction to Record Values, Ginn Press, Needham Heights, Massachusetts, 1988.

[3] M. Ahsanullah, Estimation of the parameters of the Gumbel distribution based on the m record values, Comput. Statist. Quart., 3(1900), 231-239.

[4] M. Ahsanullah, On the record values from Univariate distributions, $N a$ tional Institute of Standards and Technology Journal of Research Special Publications, 866(1993), 1-6.

[5] M. Ahsanullah, Record values. In N. Balakrishnan and A.P. Basu (eds) The Exponential Distribution: Theory, Methods and Applications, Gordon and Breach Publishers Newark, New Jersey, (1995), 279-296. 
[6] B.C. Arnold, N. Balakrishnan and H.N. Nagaraja, A First Course in Order Statistics, John Wiley and Sons, New York, 1992.

[7] B. C. Arnold, N. Balakrishnan and H. N. Nagaraja, Records, John Wiley and Sons, New York, 1988.

[8] N. Balakrishnan and M. Ahsanullah, Recurrence relations for single and product moments of record values from generalized Pareto distribution, Commun. Statist. Theor. - Meth., 23(1994), 2841-2852.

[9] N. Balakrishnan, M. Ahsanullah and P.S. Chan, On the logistic record values and associated inference, J. Appl. Statist. Sci., 2(1995), 233-248.

[10] N. Balakrishnan and P.S. Chan, Record values from Rayleigh and Weibull distributions and associated inference, National Institute of Standards and Technology Journal of Research Special Publications, 866(1993), 4151 .

[11] K. N. Chandler, The distribution and frequency of record values, J Roy. Statist. Soc. B., 14(1952), 220-228.

[12] N. L. Johnson, S. Kotz and N. Balakrishnan, Continuous Univariate Distributions, Vol. 2, Second edition, John Wiley \& Sons, New York, 1995.

[13] C.D. Lai, M. Xie and D.N. Murthy, A modified Weibull distribution, IEEE Trans. Reliab., 52(2003), 33-37.

[14] C.D. Lai, T. Moore and M. Xie, The beta model, in Proc. I. Workshops on Reliability Modeling and Analysis - From Theory to Practice, (1988),153-159.

[15] K.S. Sultan and M. E. Moshref, Record values from generalized Pareto distribution and associated inference, Metrika, 51(2000), 105-116.

[16] M. Xie, Y. Tang and T.N. Goh, A modified Weibull extension with bathtub-shaped failure rate function, Reliab. Eng. Sys. Safety, 76(2002), 279-285.

\section{Received: November 25, 2006}

